\title{
Smoking as a modifying factor in chronic rhinosinusitis: Data from the Chronic Rhinosinusitis Epidemiology Study
}

\author{
Carl Philpott ${ }^{1}$ \\ ${ }^{1}$ University of East Anglia
}

September 12, 2020

Smoking as a modifying factor in chronic rhinosinusitis

Data from the National Chronic Rhinosinusitis Epidemiology Study

Chief Investigator: Professor Carl Philpott, Professor of Rhinology \& Olfactology at University of East Anglia and Honorary Consultant ENT Surgeon, James Paget University Hospital

Mr Kristian Hutson FRCS ${ }^{1}$, Dr Allan Clark PhD ${ }^{2}$, Professor Claire Hopkins FRCS ${ }^{3}$, Mr Shahzada Ahmed FRCS ${ }^{4}$, Professor Nirmal Kumar FRCS ${ }^{5}$, Mr Sean Carrie FRCS $^{6}$, Miss Sally Erskine MRCS ${ }^{1}$, Mr Vishnu Sunkaraneni FRCS ${ }^{7}$, Professor Carl Philpott FRCS ${ }^{1,2}$

1 James Paget University Hospital NHS Foundation Trust, Gorleston, United Kingdom

${ }^{2}$ Norwich Medical School, University of East Anglia, Norfolk NR4 7TJ, United Kingdom

3Guys 8 St Thomas Hospital NHS Foundation Trust, London, United Kingdom

4 University Hospitals Birmingham NHS Foundation Trust, Birmingham, United Kingdom

5 Wrightington Wigan and Leigh NHS Foundation Trust, Wigan, United Kingdom

6Freeman Hospital, Newcastle, United Kingdom

${ }^{7}$ Royal Surrey County Hospital, Guildford, United Kingdom

On behalf of the CRES group:

Chief Investigator: Prof Carl Philpott, Professor of Rhinology \& Olfactology at University of East Anglia and Honorary Consultant ENT Surgeon, James Paget University Hospital.

Prof Carl Philpott1*§, Dr Allan Clark*, Prof Claire Hopkins2, Mr Alasdair Robertson4, Mr Shahzada Ahmed6, Mr Naveed Kara12, Mr Sean Carrie11, Mr Vishnu Sunkaraneni20, Prof Jaydip Ray17, Mr Shahram Anari7, Mr Paul Jervis10, Miss Jaan Panesaar18, Mr Amir Farboud5, Prof Nirmal Kumar3, Mr Russell Cathcart8, Mr Robert Almeyda14, Prof Hisham Khalil9, Mr Peter Prinsley13, Mr Nicolas Mansell15, Mr Mahmoud Salam16, Mr Jonathan Hobson19,

1James Paget University Hospital NHS Foundation Trust, Gorleston, 2Guys \& St Thomas' Hospital, London, 3Wrightington, Wigan \& Leigh NHS Foundation Trust, 4Southern General Hospital, Glasgow, 5Wrexham Maelor Hospital, Wales, 6University Hospitals Birmingham, 7Heart of England NHS Foundation Trust, Birmingham, 8Cumberland Infirmary, Carlisle, 9Derriford Hospital, Plymouth, 10Northampton General Hospital, 11Freeman Hospital, Newcastle, 12Sunderland Royal Infirmary, 13Norfolk \& Norwich University Hospital, 14Oxford University Hospitals, 15Royal Berkshire NHS Foundation Trust, Reading, 16The Ipswich Hospital, 17Sheffield Teaching Hospitals, 18Luton \& Dunstable Hospital, 19Warrington and Halton Hospitals NHS 
Foundation Trust, 20Royal Surrey County Hospital, Guildford, *Norwich Medical School, University of East Anglia, Norfolk NR4 7TJ, United Kingdom, §Spire Norwich Hospital.

NIHR portfolio ID: 12926; Funding: The Anthony Long Trust, the Bernice Bibby Trust .

E-mail for correspondence:C.Philpott@uea.ac.uk

This study has been reported in accordance with the STROBE statement guidelines for the reporting of observational studies.

Word count $=2893$ (main manuscript)

\section{Key points:}

1) This study sought to determine whether smoking was a risk factor for CRS and whether it has an impact on disease specific quality of life.

2) We found no significant difference in active smoking prevalence by CRS disease (CRSsNPs and CRSwNPs) vs controls. We were able however to demonstrate a significant symptom burden associated with smoking, with significantly worse SNOT-22 scores in the smoking cohort by a mean magnitude of 10 points.

3) Cigarette smoke has a deleterious effect on the quality of life and symptom burden of patients with CRS and clinicians should encourage smoking cessation alongside general CRS medical management.

\section{Abstract}

\section{Importance:}

The deleterious impact of smoking on the respiratory tract is well known, however the relationship between smoking and chronic rhinosinusitis (CRS) has not been well characterised.

Objective: We sought to analyse whether active smoking was a risk factor for CRS and whether it has an impact on disease specific quality of life.

Design: Sub-analysis of the Chronic Rhinosinusitis Epidemiology Study (CRES), a prospective, questionnaire-based case-control study conducted between October 2007 and September 2013.

Setting: Multicentre Case -Controlled across thirty UK Tertiary/ Secondary care sites.

\section{Participants:}

Participants were identified at ENT outpatient clinics and classified into CRS phenotypes as per EPOS 2012 criteria. The overall response rate of those identified to take part in the study was $66 \%$. A total of 1535 questionnaires were returned with 1470 considered eligible for inclusion.

Main Outcome(s) and Measure(s):

CRES was designed to distinguish differences in socio-economic status, geography, medical co-morbidity, lifestyle and quality of life between patients with CRS and healthy controls.

\section{Results:}

1450 patients completed the smoking question; 219 controls, 546 CRS participants without nasal polyps (CRSsNP) and 685 participants with CRS and nasal polyps (CRSwNPs+). The mean age was similar with a greater female preponderance in the control group and male in the CRSwNp group. The greatest number of active smokers was found amongst control participants (15\%) with lower rate of smokers in both CRSwNPs+ $(9.9 \%)$ and CRSsNPs patients $(13.9 \%)$ respectively $(\mathrm{p}=0.03)$. We found a significant difference in the mean difference in SNOT-22 scores between active smokers and non-smokers for both CRS phenotypes $(\mathrm{p}<0.001)$ on Analysis of Variance. In both CRS subgroups active smokers had significantly worse SNOT-22 scores 
than non-smokers by a mean magnitude of 10 points. Non smokers also demonstrated a higher percentage of surgical procedures (one or more) although this was not statistically different $(\mathrm{p}=0.098)$.

\section{Conclusions and Relevance:}

We demonstrate a significant symptom burden associated with active cigarette smoking, with significantly worse SNOT-22 scores in the smoking cohort by a mean magnitude of 10 points. We could find no strong demonstrable evidence that smoking increases the likelihood of need for revision sinus surgery.

Clinicians should encourage smoking cessation alongside general CRS medical management.

Key words : rhinosinusitis; smoking; quality of life

\section{Introduction}

\section{BACKGROUND:}

Previous population based studies including both the Global allergy \& Asthma European (GA ${ }^{2}$ LEN) survey ${ }^{1}$ and Canadian National Population Health survey ${ }^{2}$ suggest a strong association between CRS prevalence and active smoking, with a possible dose dependent association in the $\mathrm{GA}^{2} \mathrm{LEN}^{1}$ study finding a $1.5 \%$ increase in prevalence for each year smoked. Several national and international studies have also looked at smoking and its relationship to chronic rhinosinusitis (CRS); with eleven out of thirteen studies in a recent systematic review reporting increased CRS prevalence in smokers. ${ }^{3}$ Conversely a small number of studies ${ }^{4,5}$ have reported a lack of any strong association and some previous epidemiological studies have the potential to overestimate disease prevalence on methodological design. The 2000 National (England and Wales) SinoNasal Audit identified that around $20 \%$ of patients with CRS/ nasal polyps regarded themselves as active smokers, compared to a national adult smoking rate at the time of $27 \%{ }^{6}$

A number of studies have examined the possible effects of smoking on the sinonasal mucosa with variable results. This lack of consensus may result from a lack of standardisation but also highlights that a combination of different pathophysiological mechanisms may co-exist. Chistenson et $\mathrm{al}^{3}$ summarised prominent findings from available invitro and invivo studies. In vitro studies have suggested a number of possible mechanisms with smoking causing alterations in chloride ion transport,,${ }^{7,8}$ reduced mucociliary clearance ${ }^{8}$ and or reduced ciliary generation. ${ }^{9}$ In vivo results are also conflicting with possible changes in histology ${ }^{10}$ mucociliary transport ${ }^{11}$ and inflammatory cytokines ${ }^{12}$ underlying disease development. The aetiological role of the sinonasal microbiome is another topical area where there has been increasing research with respect to smoking and its potential roles in altering this microbiome and or encouraging biofilm formation. ${ }^{13}$ Some in vitro experiments have shown that repetitive exposure of tobacco smoke can promote biofilm formation within bacterial isolates from CRS patients, ${ }^{14}$ however any underlying mechanism remains poorly understood. In contrast Zhang et $\mathrm{al}^{15}$ failed to find any difference between smoking status and biofilm formation within sinus cultures taken at the time of endoscopic surgery.

With such heterogeneity in existing research no strong conclusions can currently be drawn on the exact pathophysiological mechanisms involved in CRS. Understanding the relationship of smoking to the health of sinonasal mucosa is however an important step to help direct patient care and education and may allow more accurate discussion on the likely clinical outcomes of any subsequent therapy and surgical intervention.

The Chronic Rhinosinusitis Epidemiology Study (CRES) was a prospective, questionnaire-based, case-control study conducted between October 2007 and September 2013 at thirty tertiary/secondary care sites across the United Kingdom. Patients with diagnosed CRS alongside healthy control subjects were asked to complete a single, study-specific questionnaire, capturing a variety of demographic and socio-economic variables, environmental exposures and medical co-morbidities (See appendix 1).

CRES was designed to distinguish differences in socio-economic status, geography, medical/psychiatric comorbidity, lifestyle and overall quality of life between patients with CRS and healthy controls. The specific 
aim of this analysis of the CRES database was to determine whether active smoking represents a risk factor for CRS development and/ or whether smokers experience an increased symptom burden than non-smokers. Understanding causal links will allow for more informed decision making and may clarify the potential role of smoking cessation in CRS symptom control.

\section{Methods}

The study was sponsored by the University of East Anglia (UEA) and funded by the Anthony Long and Bernice Bibby Trusts. Ethical approval was granted by the Oxford C Research Ethics Committee (Ref: 07/H0606/100).

\section{Study Design}

\section{Participants and Data Sources}

Prospective participants were identified for recruitment at ENT outpatient clinics at 30 participating centres. Patients with CRS were examined by an ENT clinician and classified into different CRS phenotypes; chronic rhinosinusitis without polyps (CRSsNPs), chronic rhinosinusitis with nasal polyps (CRSwNPs) or allergic fungal rhinosinusitis (AFRS) as per EPOS 2012 criteria $^{16}$ (see CRS participant section below). Healthy controls were recruited from family members of patients attending ENT clinics as well as members of hospital staff at recruitment sites.

Questionnaires were completed during the clinic visit or taken home to be completed and returned by prepaid post. No participant identifiable data was captured therefore consent was not required although it was implied through return of the questionnaire. Returned questionnaires were scanned and the data imported into in an electronic database in Microsoft Excel. Records in the database were compared to physical copies of the questionnaires by two members of the research team to ensure accuracy and consistency between the two.

All CRS participants and healthy controls were required to meet the inclusion/ exclusion criteria outlined below:

\section{CRS Participants}

\section{Inclusion Criteria}

Criteria for diagnosis of CRS with or without polyps (EPOS guidelines) ${ }^{16}$

At least two symptoms must be present for at least 12 weeks and include:

- One of either nasal blockage/obstruction/congestion and/or nasal discharge (anterior/posterior nasal drip)

- and either facial pain/pressure and/or reduction or loss of sense of smell

and additionally:

- endoscopic signs of polyps and/or mucopurulent discharge primarily from middle meatus and/or oedema/mucosal obstruction primarily in middle meatus

- and/or CT changes: mucosal changes within the ostiomeatal complex and/or sinuses

Patients were then classified as having CRSwNPs, CRSsNPs or AFRS. Those patients with the latter additionally adhered to either the Bent and Kuhn criteria ${ }^{17}$ or the modified Vancouver criteria. ${ }^{18}$ 


\section{Healthy Control Participants}

\section{Exclusion Criteria}

- Prior history of recurrent acute or chronic rhinosinusitis.

- Any other nose/sinus disorders e.g. allergic rhinitis (hayfever).

- Any active medical problems that have required a hospital visit within the last 12 months.

\section{Exclusion Criteria for Both Groups}

- Patients/controls unable to comprehend written English.

- Patients/controls under the age of 18 years.

\section{Quantitative Variables and Bias}

A specific question on smoking was included in the questionnaire as follows:

How much do you smoke per day (cigarettes/cigars etc.)?

Available answers were: None, $1-10,11-20$, or $>20$

\section{Statistical Methods}

Patient demographics were summarised by CRS diagnosis status using mean and standard deviation for continuous variables and the number and percentage for categorical variables. The mean SNOT-22 scores were compared between active smokers and non smokers in each CRS diagnosis group and the control group using a two sample t-test. A linear regression model was used to test if the difference in mean SNOT-22 score between active smokers and non smokers depended on the CRS subgroups using a test of interaction between CRS subgroup and smoking. No difference was detected and hence the three groups were combined into a single analysis. A linear regression model was used to adjust for potential confounding due to age, gender and a diagnosis of asthma. All analyses were conducted using Stata MP 16.0.

\section{Results}

\section{Study Participants}

A total of 1535 questionnaires were returned with 1470 considered eligible for inclusion after removal of duplicates and questionnaires with missing data (see figure 1). The overall response rate of those identified to take part in the study was $66 \%$ of those distributed. This analysis is therefore based on the 1450 participants who completed the smoking part of the questionnaire.

\section{Descriptive Data}

For the purpose of this analysis, patients with AFRS and CRSwNPs are categorised together as a single group (CRSwNPs+). As such, there were 219 controls, 546 participants with CRSsNPs and 685 participants with CRSwNPs+. The patient demographics are outlined in Table 1. With similar mean age between groups and greater female preponderance in the control group and male in the CRSwNp group.

\section{Primary Outcome Data and Main Results}

The majority of active smokers in both control and CRS groups reported smoking less than 10 tobacco products a day (63\% and $61 \%$ respectively). Comparing disease groups there appears a greater number of active smokers amongst controls (15\%), which itself was below the 2007-2009 UK national average of $21 \% .{ }^{20}$ Table 2 outlines the differences in the rates of active smokers between the three groups $(\mathrm{p}=0.039$, 
Chi-squared test) and highlights the lower rate of smokers in CRSwNPs+ participants (9.9\%) and CRSsNPs patients $(13.9 \%)$ respectively ( $\mathrm{p}=0.03$, Chi-squared test).

Mean SNOT22 scores were notably higher in the smoking cohorts for all three phenotypes. On calculating the mean difference in SNOT-22 score between active smokers and non-smokers we found a significant difference for both CRS phenotypes ( $\mathrm{p}<0.001$ on Analysis of Variance (ANOVA), see Table 3 ). In both CRSsNPs and CRSwNPs+ groups, active smokers had significantly worse SNOT-22 scores than non-smokers by a mean magnitude of 10 points. This remained significant after adjusting for age, sex and asthma (Tables 3 and 4).

Categorising CRES participants by smoking preference demonstrated a higher percentage of surgical procedures within the non-smoking cohort (Table 5), however there was no statistical difference between smoking and reporting multiple ( 1 or more) surgical procedures $(\mathrm{p}=0.098)$.

\section{Discussion}

Key results:

Unlike previous epidemiological studies we did not find any evidence of a significant difference in CRS disease status between active smokers and non-smokers $(\mathrm{p}=0.5938)$. The lower number of active smokers observed in both CRS subgroups may in part be a consequence of the higher percentage of patients with concomitant asthma diagnosis as outlined in Table 1. Active smoking appears however to have a significant impact on quality of life in both CRSsNP and CRSwNP+ phenotypes although the underlying mechanism remains debated in the common literature. Multivariable analysis has shown that the higher SNOT-22 scores demonstrated in CRS smokers remains significant even after adjusting for age, sex and asthma diagnosis (Table 4). The Minimal Clinically Importance Difference (MCID) value for SNOT-22 is 8.9, this being the smallest change in treatment outcome that an individual patient would term meaningful. Although it does not necessarily follow that smoking negates the effect of treatment, the mean higher SNOT-22 score $(>10)$ in smokers underlies the significant impact of smoking on overall symptom burden.

There was also no strong demonstrable evidence that active smoking increases the likelihood of need for revision sinus surgery although analysis of a larger cohort with standardised operative technique would help clarify this further.

\section{Interpretation:}

CRES is the largest epidemiological study of CRS in the UK to date and is the first study since the UK Sinonasal Audit to collect data on patient reported symptoms and smoking status in the context of a confirmed CRS diagnosis. The majority of previous population based studies have reported positive associations between CRS prevalence and tobacco use. ${ }^{1,2}$ The conclusions drawn by some of these studies are limited by their own methodology, as unlike CRES they relied on self-reporting of CRS diagnosis and hence are open to overestimation of true disease prevalence. Analysing the UK CRES data, we have failed to demonstrate any such positive association. We are not the first study to find a lack of association with Pilan $\mathrm{et}^{\mathrm{al}} \mathrm{l}^{5}$ in Sao Paulo finding no significant difference in CRS prevalence according to smoking status $(\mathrm{p}=0.43)$, total pack years $(\mathrm{p}=0.26)$ or following exposure to second hand smoke $(\mathrm{p}=0.18)$. Min et $\mathrm{al}^{4}$ also confirmed CRS diagnosis through physical examination but failed to find an association between active and or former smoking status and CRS prevalence. A more recent study by Lee et $\mathrm{al}^{21}$ reporting on data from the Korean Health population survey (KNHANES) found an increased CRS among active smokers however on multivariable analysis that there was no overall significant difference between CRS prevalence and the patients smoking status in those patients aged 40 years and below. They did however note a similar finding to that recorded in the European $\mathrm{GA}^{2} \mathrm{LEN}^{1}$ study that the number of years smoked is significantly associated with CRS prevalence (increasing by $1.5 \%$ for every year in total smoking period).

Some studies have suggested an increasing prevalence of CRS with total number of years smoked. ${ }^{1,21}$ The results from Caminha et $\mathrm{al}^{22}$ are however contradictory, finding on multivariable analysis that Chronic 
Obstructive Pulmonary Disease (COPD) incidence and hence a likely surrogate for greater smoking history was not associated with a higher prevalence of rhinosinusitis symptoms.

Lachanas et $\mathrm{al}^{23}$ previously demonstrated that within a general 'non-CRS' population, smokers have higher SNOT-22 scores compared to non-smokers. It is clear from the CRES data that similarly all active smokers (both active CRS and control patients) had average higher SNOT-22 scores, although this was only statistically significant for active smokers with confirmed CRS (Table 3). This adds some weight to the argument that tobacco smoke may have an adverse effect on nasal outcome measures independent to whether the patient has underlying CRS. This finding has potential implications for epidemiological studies that rely on CRS self-reporting or questionnaire-based assessments without concurrent endoscopic CRS confirmation. These studies are vulnerable to overestimating CRS complaints within the smoking population as smokers appear more likely to have QOL nasal complaints and may perceive this incorrectly as CRS.

Revision sinus surgery rates remain high in the CRS population, evidenced from the National sinonasal audit five year follow up which demonstrated increasing revision rates, reaching $19.1 \%$ at 5 years; greatest in those patient with nasal polyps $(20.6 \%)^{24}$. Previous CRES analysis demonstrated that $45 \%$ of CRS patients reported some form of surgical procedure whilst multiple surgical procedures were reported in $4 \%$ of CRSsNP patients and $23 \%$ of CRSwNP+ patients. ${ }^{25}$ Interestingly the CRES smoking cohort reported lower numbers of surgical interventions compared to non smokers (Table 5) and analysis failed to find a statistical difference between smoking status and multiple surgeries. These results suggest active smoking may not be a significant risk factor for requiring multiple surgeries, however given the nature of data collection and the low comparative number of smokers versus non smokers this may not be truly representative. There are however multiple variables that may contribute to the number of operations a patient undergoes including the level of surgeon experience and selection bias on whom to operate in which being an active smoker could play a negative factor.

Previous studies have assessed the consequence of tobacco use on symptom control and rates of revision surgery. Wu et $\mathrm{al}^{26}$ analysed revision sinus surgery rates in patients with CRSwNP and found on multivariable analysis that smokers had a significantly shorter time period (median 2.82 vs. 4.31 years) before further revision surgery was deemed necessary. A recent literature review by Reh at $\mathrm{al}^{27}$ reported conflicting evidence with respect to surgical outcomes and smoking, whilst earlier studies tended to demonstrate a deleterious effect more recent prospective studies have failed to find an similar association. These conflicting literature findings may in part be accounted for by differences in surgical intervention (e.g. polypectomy alone versus full clearance FESS) and by evolving changes in technique and instrumentation over the years. Interestingly Rudmik et $\mathrm{al}^{28}$ in their prospective study reported that active smokers with recalcitrant disease can experience similar benefits and improvement in quality of life scores following endoscopic sinus surgery as their non-smoking peers. There remains however a lack of studies looking at large numbers of high-volume smokers which may help to clarify this association further.

The CRES analysis has demonstrated a higher symptom burden in active smokers, with a mean difference in SNOT 22 scores greater than the MCID. As an observational study we are limited in our conclusions; however our failure to demonstrate an association between active smoking and higher reports of revision surgery would align with recent prospective studies concluding that surgery can be effective in smokers and should be considered as a treatment option.

\section{Limitations}

The CRES study design has certain limitations, firstly the data was self-reported and may therefore predispose to recall bias. Secondly the study only included one specific question related to current tobacco smoking, allowing us to determine whether the patient was an active smoker and if they were a mild to heavy user. The selected question did not identify whether patients were ex-smokers and did not seek to quantify 'pack year' history nor did it enquire as to the presence of other tobacco users in the household. We are therefore unable to adequately comment on whether smoking is an independent risk factor for developing CRS or comment 
on the possible role of second-hand smoke exposure in CRS prevalence. The degree of tobacco use was not evenly distributed amongst the CRES cohort with only 6-7\% of patients reported smoking heavily ( $>20$ tobacco products a day). The data must also be interpreted considering associated reporting bias relating to the quantity people reported smoking, which could be an under-representation. A further limitation of the study design meant that data collection did not allow for calculation of total years smoked, we are therefore unable to accurately comment on whether prevalence of CRS in smokers appears dose dependent.

\section{Generalisability}

CRES is a cross sectional UK based study incorporating a variety of the CRS population from across the country presenting to secondary care. The CRES study does not necessarily capture the whole CRS spectrum as mild sufferers may be managed by primary care alone and may therefore be underrepresented. Further because of the multifactorial nature of CRS it is difficult to assess the impact of one single factor on CRS pathogenesis in isolation. In contrast to other studies, CRS was diagnosed by ENT specialists according to accepted diagnostic guidelines (EPOS 2012) ${ }^{16}$, other existing studies have relied on self-diagnosis and or used different criteria making direct comparisons with the existing literature more complicated.

\section{Conclusion}

This analysis highlights the significant impact smoking has upon patient symptoms. Further studies are needed to detail the relationship between smoking and CRS subgroups to help determine causality and underlying pathophysiological mechanisms, which would enable greater intervention in these subgroups. Clinicians should be advised to encourage smoking cessation within the general CRS population but especially where symptom control is not being achieved with maximal therapy.

\section{Declarations}

\section{Ethical approval and consent to participate}

The CRES was approved by the Oxford C Research Ethics Committee (Ref: 07/H0606/100), sponsored by the University of East Anglia (UEA).

\section{Consent for publication}

Not applicable

\section{Availability of data and material}

Not applicable

\section{Funding}

The study was funded by the Anthony Long Trust (postage costs) and the Bernice Bibby Trust (research nurse time).

\section{Competing interests}

None. 


\section{Author contributions}

According to the ICMJE authorship criteria:

1. substantial contributions to conception and design of, or acquisition of data or analysis and interpretation of data

2. drafting the article or revising it critically for important intellectual content

3. final approval of the version to be published

Kristian Hutson 2, 3

Allan Clark 1, 3

Carl Philpott 1, 2, 3

Claire Hopkins 2,3

Remaining authors 3

\section{Acknowledgements}

The CRES group of otorhinolaryngologists who recruited patients to the study.

Jane Woods, Research Nurse at the James Paget University Hospital for her dedication to the study.

\section{References}

1. Hastan D, Fokkens WJ, Bachert C, et al. Chronic rhinosinusitis in Europe - an underestimated disease. A GA(2) LEN study. Allergy2011; 66 : 1216-23.

2. Chen Y, Dales R, Lin M. The epidemiology of chronic rhinosinusitis in Canadians. Laryngoscope . $2003 ; 113: 1199-205$.

3. Christensen DN, Franks ZG, McCrary HC et al. A Systematic Review of the Association between Cigarette Smoke Exposure and Chronic Rhinosinusitis. Otolaryngol Head Neck Surg. 2018;158 (5):80116.

4. Min YG, Jung HW, Kim HS, Park SK, Yoo KY. Prevalence and risk factors of chronic sinusitis in Korea: results of a nationwide survey. Eur Arch Otorhinolaryngol . 1996;253 :435-39.

5. Pilan RR, Pinna FR, Bezerra TF, et al. Prevalence of chronic rhinosinusitis in Sao Paulo. Rhinology . 2012;50:129-38.

6. Hopkins C, Browne JP, Slack R, et al. The national comparative audit of surgery for nasal polyposis and chronic rhinosinusitis. Clin Otolaryngol. 2006; $31: 390-98$.

7. Virgin FW, Azbell C, Schuster D, et al. Exposure to cigarette smoke condensate reduces calcium activated chloride channel transport in primary sinonasal epithelial cultures. Laryngoscope . 2010;120 :1465-69

8. Cohen NA, Zhang S, Sharp DB, et al. Cigarette smoke condensate inhibits transepithelial chloride transport and ciliary beat frequency. Laryngoscope . 2009;119:2269-74.

9. Tamashiro E, Xiong G, Anselmo-Lima WT, Kreindler JL, Palmer JN, Cohen NA. Cigarette smoke exposure impairs respiratory epithelial ciliogenesis. Am J Rhinol Allergy . 2009; 23 :117-22.

10. Yee KK, Pribitkin EA, Cowart BJ, et al. Smoking-associated squamous metaplasia in olfactory mucosa of patients with chronic rhinosinusitis. Toxicol Pathol . 2009;37 :594-98.

11. Tamaoki J, Taira M, Nishimura K, Nakata J, Nagai A. Impairment of airway mucociliary transport in patients with sinobronchial syndrome: role of nitric oxide. J Aerosol Med . 2000;13:239-44

12. Huang $\mathrm{CC}$, Wang $\mathrm{CH}, \mathrm{Fu} \mathrm{CH}$, et al. Association between cigarette smoking and interleukin-17A expression in nasal tissues of patients with chronic rhinosinusitis and asthma. Medicine (Baltimore) . 2016;95 (47): e5432. 
13. Ramakrishnan VR, Frank DN. Impact of cigarette smoking on the middle meatus microbiome in health and chronic rhinosinusitis. Int Forum Allergy Rhinol . 2015;5 :981-89.

14. Goldstein-Daruech N, Cope EK, Zhao KQ, et al. Tobacco smoke mediated induction of sinonasal microbial biofilms. PLoS ONE . 2011;6(1) : e15700.

15. Zhang Z, Kofonow JM, Finkelman BS, et al. Clinical factors associated with bacterial biofilm formation in chronic rhinosinusitis. Otolaryngol Head Neck Surg . 2011;144(3) :457-62.

16. Fokkens WJ, Lund VJ, Mullol J, et al. European Position Paper on Rhinosinusitis and Nasal Polyps 2012. Rhinol Suppl . 2012;23 :3-298.

17. Bent JP $3^{\text {rd }}$, Kuhn FA. Diagnosis of allergic fungal sinusitis. Otolaryngol Head Neck Surg . 1994;111(5) : 580-88.

18. Philpott CM, Javer AR, Clark A. Allergic fungal rhinosinusitis - a new staging system. Rhinology . 2011;49(3):318-23.

19. Philpott C., Erskine S., Hopkins C. et al. A case-control study of medical, psychological and socioeconomic factors influencing the severity of chronic rhinosinusitis. Rhinology .2016;54:134-40.

20. Office for National statistics. Adult smoking habits in the UK: 2009. Cigarette smoking habits among adults in the UK, including the proportion of people who smoke, demographic breakdowns, changes over time, and use of e-cigarettes. Available from: https://digital.nhs.uk/data-andinformation/publications/statistical/statistics-on-smoking/statistics-on-smoking-england-2009.

21. Lee WH, Hong SN, Kim HJ et al. Effects of cigarette smoking on rhinologic diseases: Korean National Health and Nutrition Examination Survey 2008-2011. Int Forum Allergy Rhinol .2015;5:937-43.

22. Caminha GP, Pizzichini E, Lubianca Neto JF, Hopkins C, Moreira JDS, Pizzichini MMM. Rhinosinusitis symptoms, smoking and COPD: prevalence and associations. Clin Otolaryngol.2018;43 (6):1560-65.

23. Lachanas VA, Tsea M, Tsiouvaka S, Exarchos ST, Skoulakis CE, Bizakis JG. The effect of active cigarette smoking on Sino-Nasal Outcome Test in 127 subjects without rhinologic disease. A prospective study. Clin Otolaryngol 2015;40:56-59.

24. Hopkins C, Slack R, Lund V, Brown P, Copley L, Browne J. Long-term outcomes from the English national comparative audit of surgery for nasal polyposis and chronic rhinosinusitis. Laryngoscope 2009;119 : 2459-65.

25. Philpott C, Hopkins C, Erskine S, et al. The burden of revision sinonasal surgery in the UK-data from the Chronic Rhinosinusitis Epidemiology Study (CRES): a cross-sectional study. BMJ Open2015;5 (4): e006680.

26. Wu AW, Ting JY, Platt MP, Tierney HT, Metson R. Factors affecting time to revision sinus surgery for nasal polyps: a 25-year experience.Laryngoscope. 2014;124 :29-33.

27. Reh DD, Higgins TS, Smith TL. Impact of tobacco smoke on chronic rhinosinusitis - a review of the literature. Int Forum Allergy Rhinol . 2012;2 :362-69.

28. Rudmik L, Mace J, Smith TL. Smoking and Endoscopic sinus surgery: Does smoking volume contribute to clinical outcome? Int Forum Allergy Rhinol . 2011;1 (2):145-52.

Tables

Table 1: Patient Demographics.

\begin{tabular}{llll}
\hline & Controls $(\mathbf{n = 2 1 9})$ & CRSsNP (n=546) & CRSwNP (n=685) \\
\hline Mean age & $47.29(14.91)$ & $51.78(15.31)$ & $56.00(14.50)$ \\
Gender (female) & $143(68 \%)$ & $259(53 \%)$ & $204(33 \%)$ \\
Asthma diagnosis & $22(10 \%)$ & $117(21.4 \%)$ & $336(49 \%)$ \\
\hline
\end{tabular}

Table 2. Smoking distribution and SNOT-22 scores by group

\begin{tabular}{llllll}
\hline Disease status & Number of patients (n) & Number of smokers & \% & Mean Snot-22 & SD \\
\hline Controls & 219 & 33 & 15.1 & 12.11 & 13.95
\end{tabular}




\begin{tabular}{llllll}
\hline Disease status & Number of patients $(\mathbf{n})$ & Number of smokers & \% & Mean Snot-22 & SD \\
\hline CRSsNPs & 546 & 76 & 13.9 & 45.67 & 21.05 \\
CRSwNPs & 685 & 68 & 9.9 & 44.41 & 21.62 \\
\hline
\end{tabular}

Table 3. Difference in mean SNOT-22 scores by smoking status and CRS phenotype.

\begin{tabular}{|c|c|c|c|c|c|}
\hline Disease status & Mean SNOT-22 (Non-smokers) & SD & Mean SNOT-22 (Smokers) & SD & Me \\
\hline Controls & 11.23 & 13.08 & 16.82 & 17.77 & \\
\hline CRSsNPs & 44.35 & 21.02 & 54.66 & 18.99 & 10 \\
\hline CRSwNPs & 43.47 & 21.25 & 53.64 & 24.14 & 10 \\
\hline Overall (CRS + Controls) & 39.22 & 23.18 & 47.58 & 25.31 & 8 . \\
\hline
\end{tabular}

Table 4: Mean differences in SNOT-22 scores adjusting for age, gender and asthma.

\begin{tabular}{lll}
\hline Model & Mean difference (95\% CI) & p- value \\
\hline Age and Gender & $7.53(3.22,11.84)$ & 0.001 \\
Age, Gender, Asthma & $8.56(4.31,12.81)$ & $<0.001$ \\
Age, Gender, Asthma, CRS diagnosis & $8.38(4.72,11.93)$ & $<0.001$ \\
\hline
\end{tabular}

Table 5: Number of reported surgical procedures between smokers and Non smokers.

\begin{tabular}{|c|c|c|c|}
\hline Variable & Non smoker $(n=1273)$ & Smoker $(n=177)$ & Odds ratio $(95 \% \mathrm{CI})$ \\
\hline Previous Sinonasal surgery & $503(39.6 \%)$ & $51(28.8 \%)$ & $0.62(0.44,0.87)$ \\
\hline Previous sinus surgery (ESS) & $156(12.6 \%)$ & $13(7.6 \%)$ & $0.57(0.31,1.02)$ \\
\hline Previous nasal polypectomy (ENP) & $302(26.8 \%)$ & $20(13 \%)$ & $0.41(0.25,0.66)$ \\
\hline Multiple ESS/ENP & $144(12.6 \%)$ & $13(8.1 \%)$ & $0.61(0.34,1.10)$ \\
\hline
\end{tabular}

Figure Legends

Figure 1. Participant flow diagram 


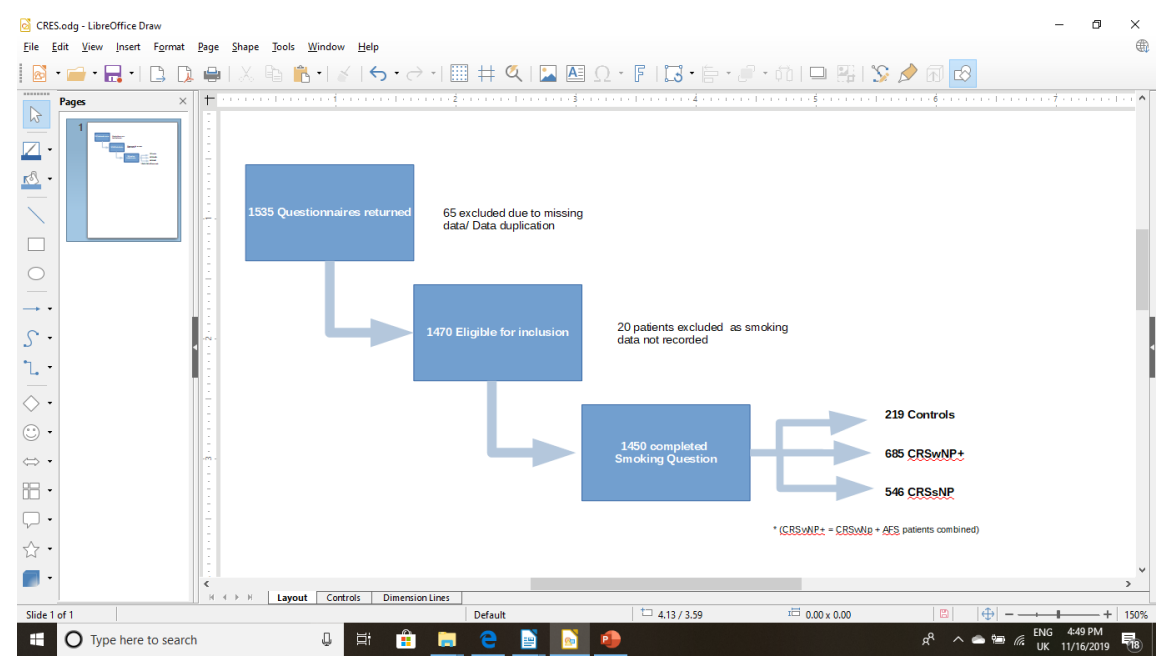

Appendix 1: Study questionnaire 
-

Ret. $\square \square \square \square$

Please try to fill in ALL parts of the questionnaire, even if you do not have sinus problems and do not feel they are directly relevant to you.

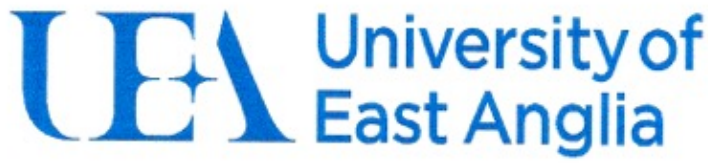

\section{CHRONIC RHINOSINUSITIS EPIDEMIOLOGY STUDY (CRES)}

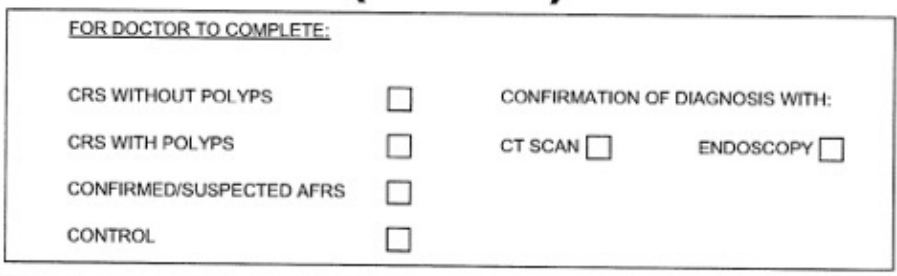

RECRUITMENT SITE

JPUH $\square$

LDH $\square$

Cl $\square$

IRH $\square$

OUH $\square$

$\mathrm{RCH} \square$

DBH $\square$
NNUH $\square$
RSCH $\square$
SRI $\square$
HEFT $\square$
SAMBU $\square$
RGH $\square$
Other $\square$
WWL

GUYS

SGH $\square$

QEH

СTHB

AUHNT

ther, please specify
SPIRE

QMC

BCUH

STH

WHH

RBNFT

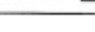

NGH $\mathrm{FH}$ RAH $\square$ WI PHNT $\square$ HWPH $\square$

Please return the questionnaire to the Norwich Modical School, UEA, Nonwich - for the attention of Mr Carl Philpott 